\title{
Microemulsion-based Drug Delivery System for Enhancement of Absorption Efficiency of Levofloxacin Hemihydrate
}

\author{
Nadeem Kizilbash \\ Department of Medical Laboratory Technology, Faculty of Applied Medical Sciences, Northern \\ Border University, Arar-91431, Saudi Arabia
}

\begin{abstract}
The possibility of enhancement of absorption efficiency of Levofloxacin Hemihydrate, a synthetic quinolone antibiotic, was explored by the use of water-in-oil microemulsion system. This system was selected because of its higher reported membrane permeability according to both in vitro and in vivo studies. A microemulsion comprising tween 20/1-propanol/water and clove oil was developed and characterized by conductivity measurements, viscosity measurements and refractive index measurements. It was found that loading of the drug had no negative effect on the stability of the system. Measurement of the partition coefficient suggests that Levofloxacin hemihydrate is present in water droplets as well as in the continuous oil phase and may also be present at the interface.
\end{abstract}

Keywords: Drug delivery, Levofloxacin Hemihydrate, microemulsion system, nanoparticles, ternary phase diagram.

\section{INTRODUCTION}

Due to the poor bioavailability through gastrointestinal (GI) tract, the oral delivery of therapeutic drugs has been a challenge for pharmacologists. Various strategies have been adopted during the recent decades to develop effective oral drug delivery systems like chemical modification [1], encapsulation into particulate delivery systems [2], preparation of macromolecular conjugates [3] and targeted delivery to colon. However, none of these efforts could be considered as a breakthrough based on currently available data. Microemulsion systems have been studied for the absorption enhancement for many years due to some prominent advantages such as thermodynamic stability, protection effect against intestinal enzymes, and high dispersion property [4, 5]. Among various microemulsion systems, oil-in-water $(\mathrm{o} / \mathrm{w})$ microemulsion system is considered more promising because it can improve the oral bioavailability of hydrophobic drugs such as cyclosporine [6]. On the other hand, some reports have suggested that the water-in-oil (w/o) microemulsion has a significant potential to increase permeability of hydrophilic drugs across the intestinal mucosa and thus enhance their oral bioavailability $[7,8]$.

Generally, there are three types of microemulsion: water-in-oil (w/o), bicontinuous and oil-in-water $(\mathrm{o} / \mathrm{w})[\mathbf{9}, \mathbf{1 0}]$. Some advantages of microemulsions as a drug delivery system are their thermodynamic stability, ease of preparation, ease of sterilization and improvement in solubility of almost all types of drugs. According to literature, it is also clear that the same microemulsion can carry both lipophilic and hydrophilic drugs. Enhancement in absorption due to surfactant induced permeability and improvement in the efficiency of the drug make microemulsion very useful as drug delivery systems [11].

Levofloxacin Hemihydrate was selected as a model drug (Figure 1) and a pseudo-ternary system comprising water, tween 20,1-propanol and clove oil was chosen. Clove oil possesses a number of therapeutic properties such as being an analgesic, antiseptic, antispasmodic, anti-neuralgic, carminative, anti-infectious, disinfectant and insecticidal substance. It is beneficial to the digestive system and effective against nausea, diarrhea, flatulence, spasms and parasites, as well as bad breath. The surfactant used in this work exhibits excellent biocompatibility [10]. The pseudo-ternary phase diagram has been delineated for the chosen system at a constant surfactant/co-surfactant ratio. The changes in the internal structure of both non-loaded and drug-loaded microemulsion samples were monitored by different experimental techniques like conductivity measurements, viscosity and UVVis. spectroscopy to explore the possibility of enhancement of absorption efficiency of Levofloxacin Hemihydrate by the use of this water-in-oil microemulsion system. 
<smiles>C[C@@H]1COc2c(N3CCN(C)CC3)c(F)cc3c(=O)c(C(=O)O)cn1c23</smiles>

Figure1. Chemical structure of Levofloxacin Hemihydrate

\section{MATERIALS AND MethodS}

\subsection{Chemicals}

Tween 20 was purchased from BDH Laboratory (England), 1-propanol was obtained from Merk (Germany), Levofloxacin hemihydrate was purchased from Zhejiang chemical (China) and clove oil was purchased from local market. Water used in all experiments was doubly-distilled and deionized.

\subsection{Preparation of Microemulsion System}

The microemulsion samples were selected from two dilution lines in the microemulsion region of the phase diagram. The surfactant and co-surfactant were added in a 1:1 volume ratio to obtain a surfactant mixture. Clove oil was then added and the mixture was allowed to homogenize for 5 minutes, and then the required amount of water was added. These components were mixed using a hot plate with a magnetic stirrer (Corning, model PC-420D) for 10 minutes at room temperature to maintain the homogeneity of the system. A sonicator (Elam E 30H) was also used for homogenizing and mixing of the constituents. All samples were stored at room temperature for at least 48 hours to achieve equilibrium before further experimentation. In the first type of dilution line, the concentration of surfactant mixture was kept constant $64.8 \%$ while the ratio of water to clove oil varied between $0 \%$ and $35.2 \%$. In the second type of dilution line, one sample was selected from microemulsion region with minimum water concentration, and then it was titrated against water using micropipette at constant temperature. A microemulsion sample was further selected, for detailed investigation of the incorporation of drug in the microemulsion system.

\subsection{Centrifugation}

Stability of microemulsion samples was tested by carrying out centrifugation at $6000 \mathrm{rpm}$ for 10 minutes by use of a Hermle Z200 centrifuge. Table 1 summarizes the physical parameters of pure microemulsion sample ME-A, and after incorporation of the drug, Levofloxacin hemihydrate.

Table1. Physical parameters of pure microemulsion sample ME-A, and after incorporation of the drug, Levofloxacin hemihydrate.

\begin{tabular}{|c|c|c|c|}
\hline Physical Property & Pure ME-A & $\mathbf{( 0 . 5 \% )}$ drug-loaded ME-A & $\mathbf{( 1 \% ) \text { drug-loaded ME-A }}$ \\
\hline Conductivity $(\mu \mathrm{S} / \mathrm{cm})$ & 37.8 & 39.3 & 42.1 \\
\hline Viscosity $(\mathrm{cP})$ & 96.5 & 97.7 & 99 \\
\hline
\end{tabular}

\subsection{Viscosity Measurements}

Viscosities of microemulsion samples were measured by digital viscometer (Brookfield DV-E) with spindle number 62. Time of flow of the selected non-loaded and drug-loaded microemulsion samples was measured. For each measurement, the spindle was washed, rinsed and dried. The temperature was kept constant at $25^{\circ} \mathrm{C}$.

\subsection{Conductivity Measurements}

The electrical conductivity was measured by means of a Microprocessor Conductivity Meter (WTW82362Weilheim) fitted with an electrode (WTW06140418) having a cell constant of $1 \mathrm{~cm}^{-1}$. A thermostat (Julabo) was used to maintain the temperature at $25^{\circ} \mathrm{C}$. 


\subsection{Refractive Index Measurements}

In the present study the refractive index of the selected microemulsion systems were determined by using ATAGO RX-5000 Refractometer at 25 0C. A thermostat (Grant Instruments Ltd.) with circulating water was attached to maintain the temperature in the required range.

\section{RESUlTS AND DisCuSSION}

\subsection{Pseudoternary Phase Diagram}

Figure 2 shows the constructed pseudoternary phase diagram with the area inside the frame indicating the microemulsion region while the area outside the frame indicating a turbid region containing multiphase systems. It should be noted that the area of microemulsion region is considerably large since 1-propanol acts as a co-surfactant and interacts with the surfactant monolayer to increase the flexibility of the interfacial film. Microemulsions are formed spontaneously when their components come together [12]. The order of mixing of various components is not expected to influence the formation of microemulsions if the system is indeed thermodynamically stable. It is the low interfacial tension of microemulsions that favors the formation of a thermodynamically stable dispersion [13].

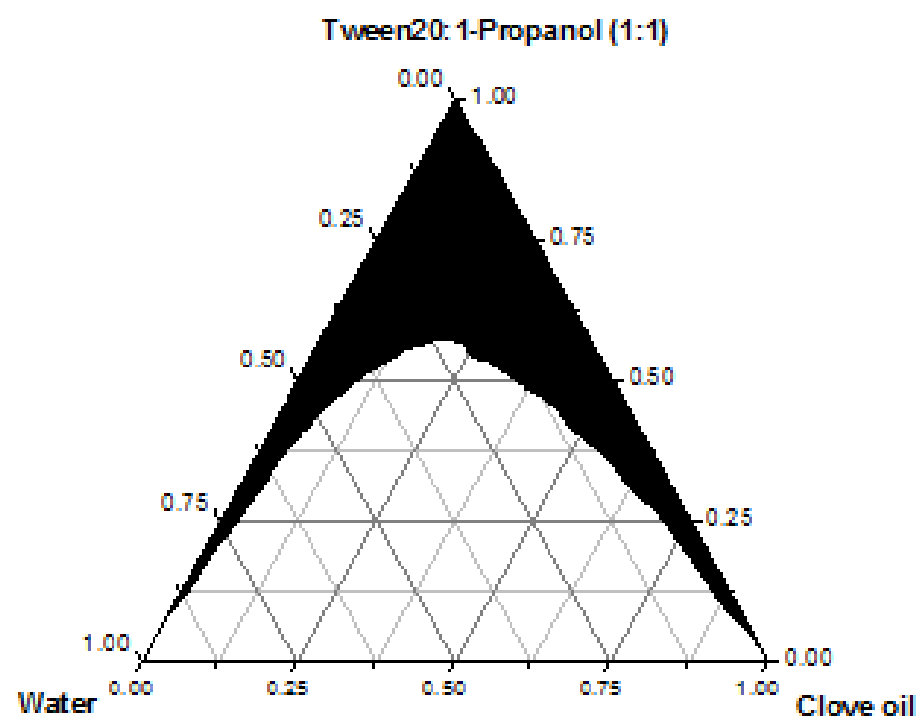

Figure2. The pseudoternary phase diagram showing microemulsion region of tween 20/1-propanol/clove oil/water

\subsection{Conductivity Values along Dilution Line XY}

The results of variation of conductivity along the dilution line XY (Figure 3) as a function of water content (vol \%) are shown in Figure 4. This behavior exhibits a profile characteristic of percolative conductivity $[\mathbf{1 4}, \mathbf{1 5}]$. The conductivity is initially low in oil-surfactant mixture but increases with increase in aqueous phase. The low conductivity below $27 \%$ water contents suggests that the reverse phase droplets (isolated droplets in a non-conducting clove oil medium, forming w/o microemulsion) are discrete and have little interaction. When the water content was raised to $27 \%$, the values of conductivity increased linearly up to a value of about $60 \%$. At high water content, the interaction among the aqueous domains becomes increasingly important and water forms a network of conductive channels (bicontinuous phase). With further increase in water content above $60 \%$, the conductivity decreases sharply. The system becomes turbid which contributes to the dilution of $\mathrm{o} / \mathrm{w}$ microemulsion where added water decreases the concentration of discrete oil droplets [16]. The change in the slope of conductivity can be interpreted, as a structural transition to bicontinuous system from w/o microemulsion [17], nearly at $27 \%$ water contents. This transition takes place once the aqueous phase becomes continuous phase. This is in line with the observation made in phase study. Thus, the conductivity versus water contents plots illustrates occurrence of three different structures (namely w/o, bicontinuous and o/w). It was also noted that Levofloxacin-hemihydrate does not affect the microstructures of the microemulsion (Figure 5). 


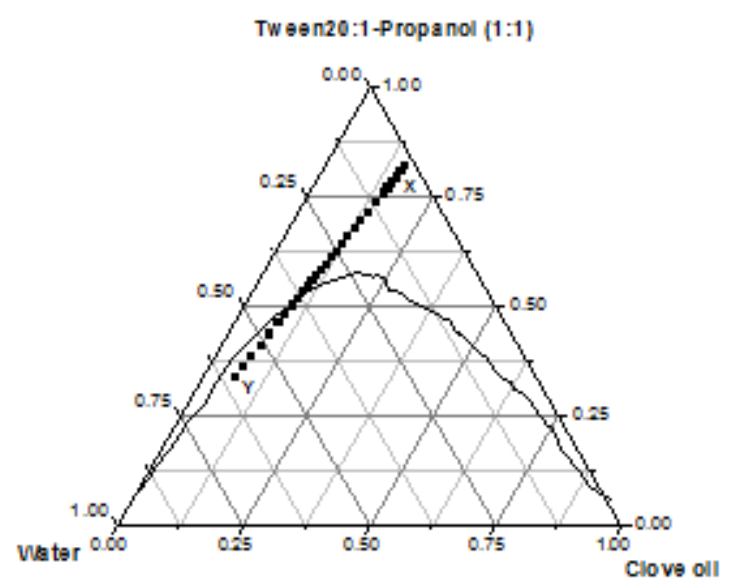

Figure3. The pseudoternary phase diagram showing the dilution line XY

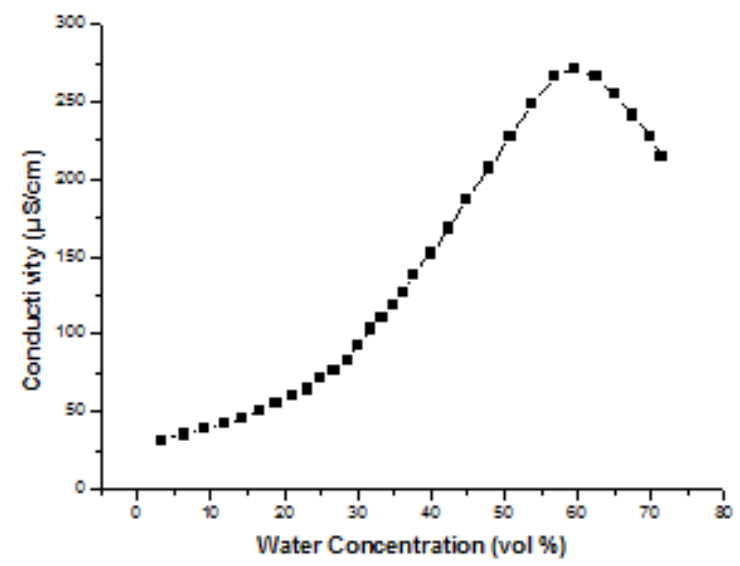

Figure4. Variation in electrical conductivity with water concentration along the dilution line XY

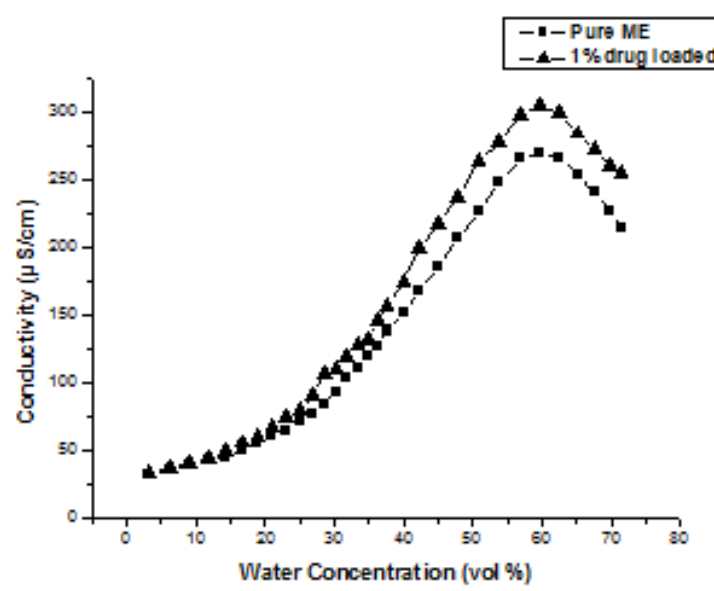

Figure5. Comparison of electrical conductivity of pure and $1 \%$ Levofloxacin hemihydrate-loaded microemulsion samples along the dilution line $X Y$

\subsection{Conductivity Values along Dilution Line GH}

The relationship between water concentrations and conductivity along dilution line GH (Figure 6) is shown in Figure 7. The conductivity of the studied samples was low as long as the water concentration was smaller than $13 \%(\mathrm{v} / \mathrm{v})$. Above $13 \%$ water concentration, a drastic increase in conductivity was observed. It was due to the transition of oil continuous microemulsion to bicontinuous microemulsion. A further sharp increase in conductivity was found when water concentration reaches $29 \%$. This increment in conductivity was most likely caused by a transition from bicontinuous microemulsion to a water continuous microemulsion system [18]. A slight increase in conductivity was noted after loading drug (Figure 8), this increment is derived from the Levofloxacin hemihydrate. 


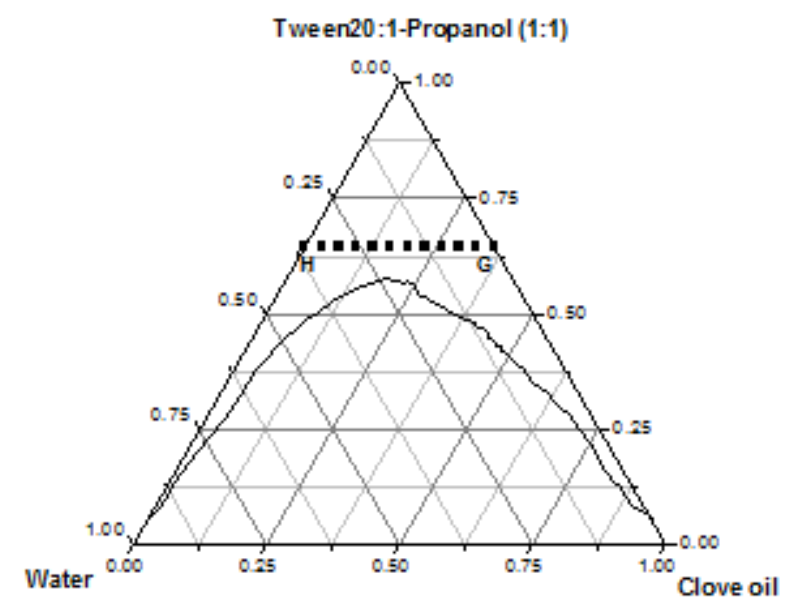

Figure6. The pseudoternary phase diagram showing the dilution line GH

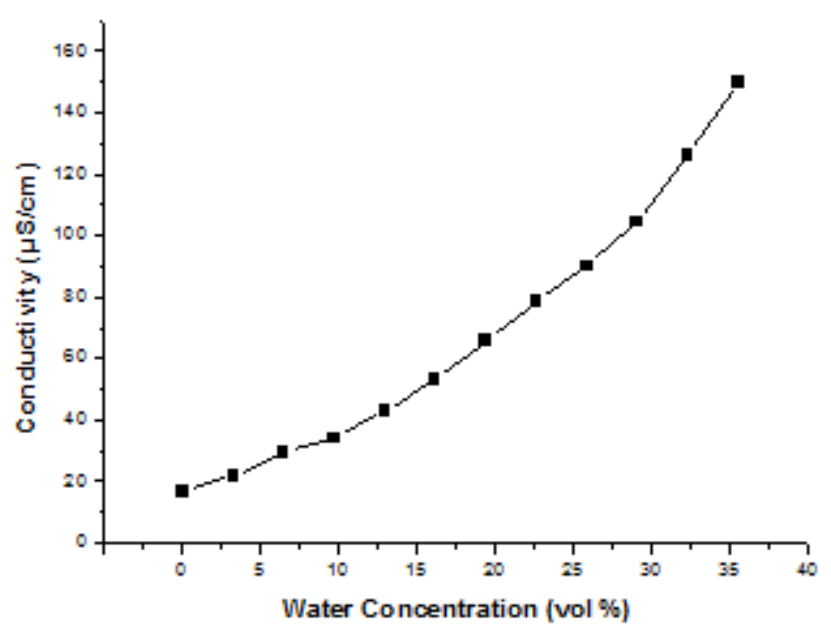

Figure7. Electrical conductivity as a function of the water concentration of the systems along dilution line GH

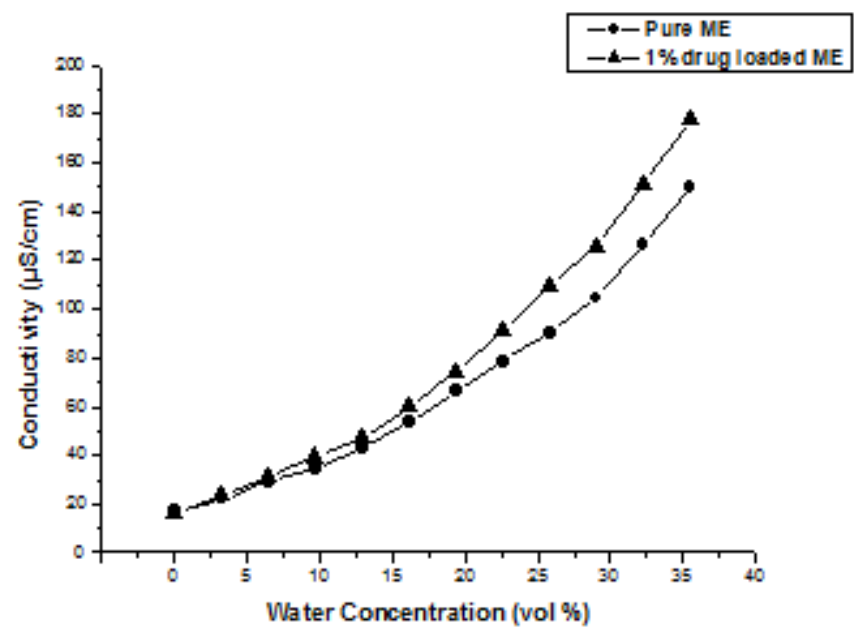

Figure8. Comparison of electrical conductivity of pure and $1 \%$ Levofloxacin hemihydrate- loaded microemulsion samples as a function of the water concentration along dilution line GH

\subsection{Viscosity Values along Dilution Line XY}

The water concentration increased from $3 \%$ to $27 \%$ (v/v) and the viscosity rose from $31.7 \mathrm{cP}$ to 37.3 $\mathrm{cP}$. The rise in viscosity in interpreted to be due to the increase in the size of dispersed particles which increases the electrostatic interactions in the dispersed phase. It was noted that further increase in concentration of water from $27 \%$ to $60 \%$ causes a decrease in viscosity very slowly, in other words viscosity remains relatively constant. In this region, the decrease in viscosity from $37.3 \mathrm{cP}$ to $31 \mathrm{cP}$ 
and this behavior is typical for bicontinuous or solution-type microemulsion system [19]. When the concentration of water increased from $60 \%$, an abrupt decrease in viscosity from $31 \mathrm{cP}$ to $16.5 \mathrm{cP}$ was noted. This change in trend is most likely due to the transformation of bicontinuous to $\mathrm{o} / \mathrm{w}$ microemulsion (Figure 9). Further, 1\% Levofloxacin hemihydrate-loaded microemulsion system has somewhat higher viscosities than its pure counterparts (Figure 10). But there is no structural effect on the microstructure of the microemulsion system.

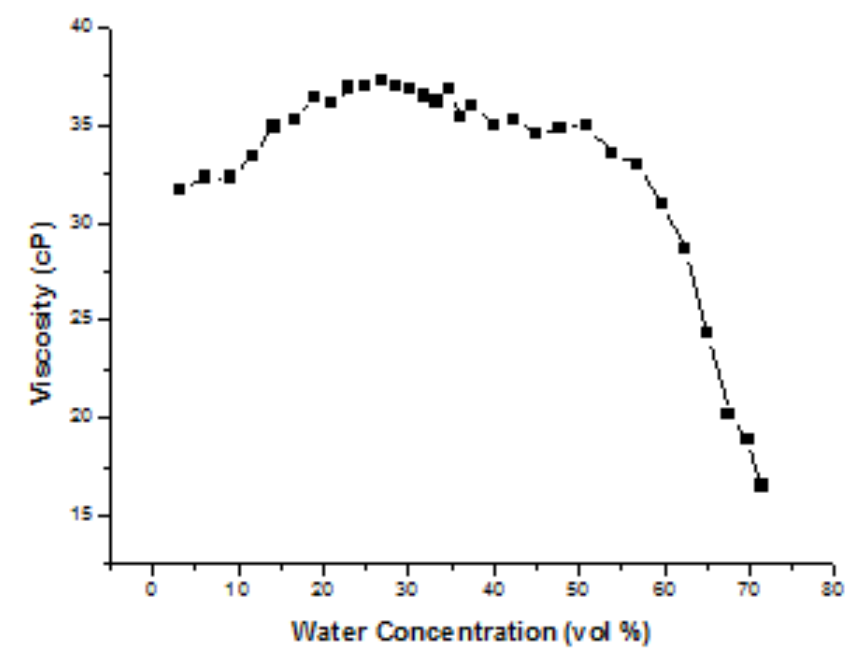

Figure9. Variation of viscosity with water concentration along dilution line XY

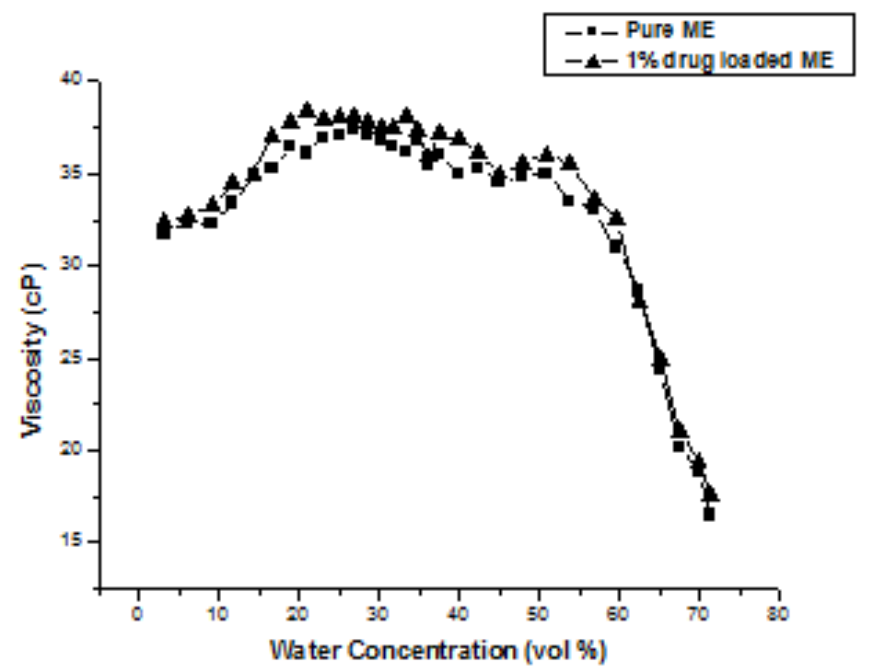

Figure10. Comparison of viscosity of pure and $1 \%$ Levofloxacin hemihydrate-loaded microemulsion samples along the dilution line $X Y$

\subsection{Viscosity Values along Dilution Line GH}

The viscosity versus water contents graph (Figure 11) along dilution line GH shows a similar trend as that of viscosity graph of dilution line XY (Figure 4). Initially viscosity increased from $29 \mathrm{cP}$ to 34.3 $\mathrm{cP}$ with increase in water concentration from 0 to $13 \%(\mathrm{v} / \mathrm{v})$. It is well known that an increase in the volume fraction of the dispersed phase in microemulsion causes an increase in viscosity [20]. Viscosity changes reflect a transformation of microstructure as a function of water concentration. This initial increment of viscosity with increase of water concentration is probably the consequence of attractive interaction and aggregation of droplets of water phase including molecular reorganization on the interface. Measured values of viscosity of these samples decreased from $34.3 \mathrm{cP}$ to $32.9 \mathrm{cP}$ with increase in water phase concentration from $13 \%$ to $29 \%(\mathrm{v} / \mathrm{v})$. The decrease in viscosity is relatively slow. This behavior is typical for bicontinuous or possibly solution type microemulsion [20]. Finally, the curve of viscosity versus volume fraction of water decreased abruptly from $32.9 \mathrm{cP}$ to $25 \mathrm{cP}$ with increasing water content to $29 \%(\mathrm{v} / \mathrm{v})$. This is most probably due to the transformation of bicontinuous microemulsion to water continuous system. $1 \%$ Levofloxacin hemihydrate loaded samples had no significant effect on viscosity (Figure 12). 


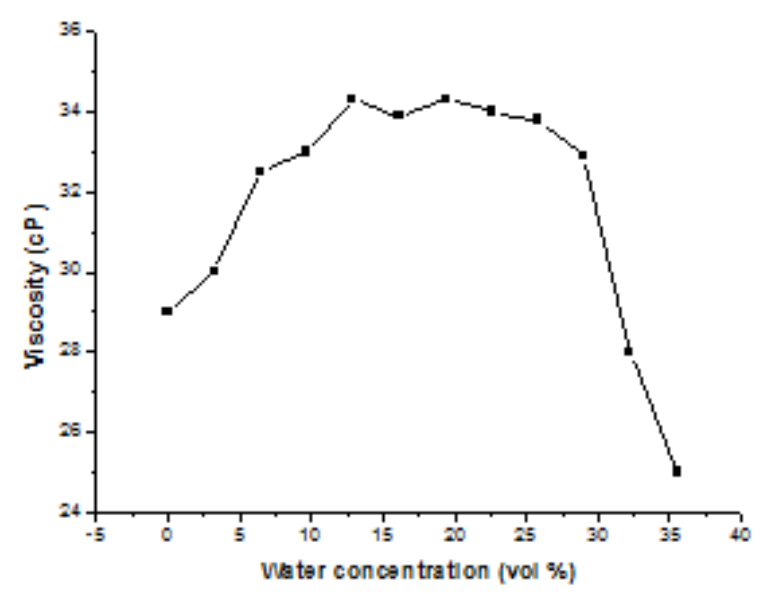

Figure11. Viscosity as a function of the water concentration of the systems along the dilution line GH

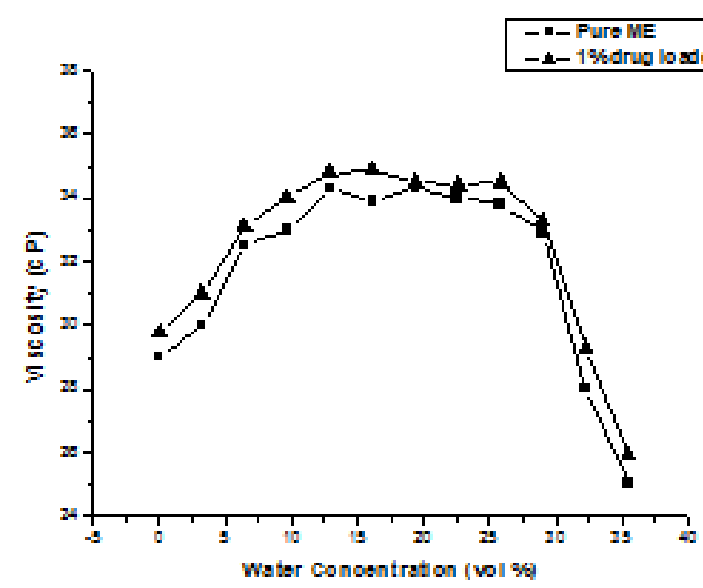

Figure12. Comparison of viscosity of pure and 1\% Levofloxacin hemihydrate-loaded microemulsion samples as a function of the water concentration along the dilution line $\mathrm{GH}$

\subsection{Refractive Index Values along Dilution Line GH}

Refractive index values of all the pure microemulsion samples and their pure components were noted [21]. These values are intermediate between the values of pure components. $1 \%$ Levofloxacin hemihydrate-loaded systems showed a little increase in values of refractive index. The increment is almost the same in both the pure and drug-loaded microemulsion samples showing that the solubility is almost the same (Figure 13). From these results, we can also conclude that drug is almost equally soluble in both oil and water phase.

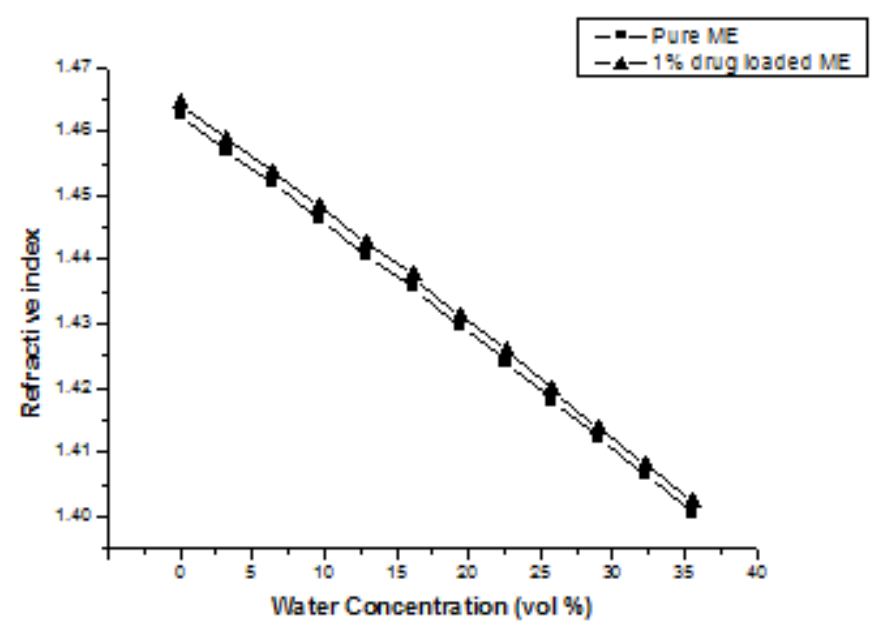

Figure13. The Comparison in refractive index values of pure and their 1\% Levofloxacin-hemihydrate-loaded microemulsion samples along dilution line $G H$ 


\section{Conclusion}

Levofloxacin hemihydrate can be effectively incorporated into a w/o microemulsion system consisting of tween20/1-propanol/water and clove oil. The proper physicochemical characteristics have been demonstrated for the newly formulated system. It was found that $1 \%(\mathrm{w} / \mathrm{w})$ Levofloxacin hemihydrate had no any effect on either microstructure of the microemulsion system nor on its percolation behavior. Refractive index measurements showed that all the samples possess almost the same drug solubility. The prepared w/o microemulsion system can potentially be used after modifications to make it more biocompatible, as an effective drug delivery system for hydrophilic bioactive molecules such as Levofloxacin hemihydrate.

\section{REFERENCES}

[1] Kipnes M, Dandona P, Tripathy D, Still JG, Kosutic G. Control of postprandial plasma glucose by an oral insulin product (HIM2) in patients with type 2 diabetes. Diabetes Care. 2003; 26(2):421-6.

[2] Van Der Lubben IM, Konings FA, Borchard G, Verhoef JC, Junginger HE. In vivo uptake of chitosan microparticles by murine Peyer's patches: visualization studies using confocal laser scanning microscopy and immunohistochemistry. Journal of Drug Targeting. 2001;9(1):39-47.

[3] Russell-Jones GJ. Use of targeting agents to increase uptake and localization of drugs to the intestinal epithelium. Journal of Drug Targeting. 2004 Feb 1;12(2):113-23.

[4] Spernath A, Aserin A. Microemulsions as carriers for drugs and nutraceuticals. Advances in Colloid and Interface Science. 2006;128:47-64.

[5] Jadhav KR, Shaikh IM, Ambade KW, Kadam VJ. Applications of microemulsion based drug delivery system. Current Drug Delivery. 2006;3(3):267-73.

[6] Ritschel WA. Microemulsion technology in the reformulation of cyclosporine: the reason behind the pharmacokinetic properties of Neoral. Clinical Transplantation. 1996;10(4):364-73.

[7] Constantinides PP, Scalart JP, Lancaster C, Marcello J, Marks G, Ellens H, Smith PL. Formulation and intestinal absorption enhancement evaluation of water-in-oil microemulsions incorporating medium-chain glycerides. Pharmaceutical Research. 1994;11(10):1385-90.

[8] Prasad YR, Puthli SP, Eaimtrakarn S, Ishida M, Yoshikawa Y, Shibata N, Takada K. Enhanced intestinal absorption of vancomycin with Labrasol and D- $\alpha$-tocopheryl PEG 1000 succinate in rats. International Journal of Pharmaceutics. 2003;250(1):181-90.

[9] Paul BK, Moulik SP. Microemulsions: an overview. Journal of Dispersion Science and Technology. 1997;18(4):301-67.

[10] Lawrence MJ, Rees GD. Microemulsion-based media as novel drug delivery systems. Advanced Drug Delivery Reviews. 2000;45(1):89-121.

[11] Vyas SP, Khar RK. Submicron emulsion. Targeted and Controlled Drug Delivery Novel Carriers Systems. CBS Publishers and Distributors. 2002:291-4.

[12] Osborne DW, Middleton CA, Rogers RL. Alcohol-free microemulsions. Journal of Dispersion Science and Technology. 1988;9(4):415-23.

[13] Grainger DW. Controlled release of drugs: Polymers and aggregate systems. Edited by M. Rosoff, VCH Verlagsgesellschaft, Weinheim, 1989, xi, 315 pp., bound, DM 132.-ISBN 3-527-26797-2. Advanced Materials. 1989;1(8-9):309-11.

[14] Mehta SK, Bala K. Tween-based microemulsions: a percolation view. Fluid Phase Equilibria. 2000;172(2):197-209.

[15] Grest GS, Webman I, Safran SA, Bug AL. Dynamic percolation in microemulsions. Physical Review A. 1986;33(4):2842.

[16] Podlogar F, Gašperlin M, Tomšič M, Jamnik A, Rogač MB. Structural characterisation of waterTween $40 \AA / I m w i t o r ~ 308 ®-i s o p r o p y l$ myristate microemulsions using different experimental methods. International Journal of Pharmaceutics. 2004;276(1):115-28.

[17] Lv FF, Zheng LQ, Tung CH. Phase behavior of the microemulsions and the stability of the chloramphenicol in the microemulsion-based ocular drug delivery system. International Journal of Pharmaceutics. 2005;301(1):237-46. 
[18] El-Laithy HM. Preparation and physicochemical characterization of dioctyl sodium sulfosuccinate (aerosol OT) microemulsion for oral drug delivery. AAPS PharmSciTech. 2003;4(1):80-9.

[19] Alany RG, Tucker IG, Davies NM, Rades T. Characterizing colloidal structures of pseudoternary phase diagrams formed by oil/water/amphiphile systems. Drug Development and Industrial Pharmacy. 2001; 27(1):31-8.

[20] Bennett KE, Hatfield JC, Davis HT, Macosko CW, Scriven LE. Viscosity and conductivity of microemulsions. In Microemulsions 1982 (pp. 65-84). Springer US.

[21] Ghosh PK, Majithiya RJ, Umrethia ML, Murthy RS. Design and development of microemulsion drug delivery system of acyclovir for improvement of oral bioavailability. AAPS PharmSciTech. 2006; 7(3):E172-7.

\section{AUTHOR'S BIOGRAPHY}

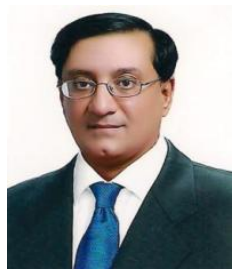

Dr. Nadeem Kizilbash, received his Bachelor's degree in Chemistry in 1989 from Longwood College in Farmville, U.S.A. His Master's degree was also in Chemistry in 1994 from Washington University in St. Louis, U.S.A. His doctoral degree was awarded in Biophysics in 2003 from Boston University in U.S.A. He is presently working as an Assistant Professor of Biochemistry at Northern Border University in Arar, Saudi Arabia. He has previously worked as an Assistant Professor at Quaid-iAzam University in Pakistan. His work experience also includes service as a Senior Scientist at National Institute for Biotechnology and Genetic Engineering in Pakistan. He has published thirty five papers in various journals on topics as diverse as Proteomics, Protein Structure, Drug Delivery and Gene Polymorphism. 